\title{
Aspergillus fumigatus responds to natural killer (NK) cells with upregulation of stress related genes and inhibits the immunoregulatory function of NK cells
}

\author{
Andreas Schneider ${ }^{1}$, Michael Blatzer ${ }^{2}$, Wilfried Posch $^{2}$, Ralf Schubert ${ }^{3}$, Cornelia \\ Lass-Flörl' ${ }^{2}$, Stanislaw Schmidt ${ }^{1, *}$ and Thomas Lehrnbecher ${ }^{1, *}$ \\ ${ }^{1}$ Division of Pediatric Hematology and Oncology, Hospital for Children and Adolescents, Johann Wolfgang Goethe-University, \\ Frankfurt, Germany \\ ${ }^{2}$ Division of Hygiene and Medical Microbiology, Medical University of Innsbruck, Innsbruck, Austria \\ ${ }^{3}$ Division of Pediatric Pulmonology, Allergology and Cystic Fibrosis, Hospital for Children and Adolescents, Johann Wolfgang \\ Goethe-University, Frankfurt, Germany \\ * Co-senior authors
}

Correspondence to: Thomas Lehrnbecher, email: Thomas.Lehrnbecher@kgu.de

Keywords: Aspergillus fumigatus, human natural killer cell, gene expression, perforin, cyłokine, Immunology and Microbiology Section, Immune response, Immunity

Received: September 02, 2016 Accepted: September 17, $2016 \quad$ Published: October 12, 2016

\section{ABSTRACT}

Natural Killer (NK) cells are active against Aspergillus fumigatus, which in turn is able to impair the host defense. Unfortunately, little is known on the mutual interaction of NK cells and A. fumigatus. We coincubated human NK cells with $A$. fumigatus hyphae and assessed the gene expression and protein concentration of selected molecules. We found that $A$. fumigatus up-regulates the gene expression of pro-inflammatory molecules in NK cells, but inhibited the release of these molecules resulting in intracellular accumulation and limited extracellular availability. $A$. fumigatus down-regulated mRNA levels of perforin in NK cells, but increased its intra- and extracellular protein concentration. The gene expression of stress related molecules of $A$. fumigatus such as heat shock protein hsp90 was up-regulated by human NK cells. Our data characterize for the first time the immunosuppressive effect of $A$. fumigatus on NK cells and may help to develop new therapeutic antifungal strategies.

\section{INTRODUCTION}

Natural Killer (NK) cells are lymphocytes of the innate immune system which are able to kill their target by cytotoxic molecules such as perforin or granzyme $\mathrm{B}$ or by death receptor-mediated apoptosis. In addition, NK cells enhance the activity of other arms of the host immune response such as of professional phagocytes, dendritic cells, and $\mathrm{T}$ cells through chemokines and cytokines such as interferon (IFN)- $\gamma$ and granulocyte-macrophage colonystimulating factor (GM-CSF) [1]. Although the term of NK cells came from their natural ability to kill tumor cells, it has been demonstrated that NK cells also exhibit cytotoxicity against virus-infected cells and bacteria, and play an important role in the antifungal host response such as against Aspergillus fumigatus, which is a major cause for morbidity and mortality in patients receiving chemotherapy for cancer or undergoing hematopoietic stem cell transplantation [2]. For example, it has been demonstrated that in neutropenic mice with invasive aspergillosis, depletion of NK cells increased the fungal load, whereas the transfer of activated NK cells resulted in greater pathogen clearance from the lungs [3]. From the pathogen's point of view, A. fumigatus produces a variety of proteins, such as proteinases or mycotoxins, all of which are important in the defense mechanisms of the fungus and tissue invasion, as they detoxify antifungal molecules produced by the host or break down the host defense barriers [4]. As we have demonstrated in an earlier study that A. fumigatus decreases the protein concentration of NK cell-derived IFN- $\gamma$ in the supernatant [5], we investigated the influence of A. fumigatus on the expression of selected genes which are known to play a role in the antifungal activity of NK cells. Given the complex nature of the host-pathogen interaction, we also assessed the response of $A$. fumigatus to host immunity. 


\section{RESULTS}

\section{A. fumigatus increases mRNA levels of proinflammatory cytokines, but causes intracellular accumulation of these molecules and limits extracellular availability}

When IL-2 prestimulated NK cells were incubated with A. fumigatus hyphae, mRNA levels of the proinflammatory molecule IFN- $\gamma(I F N G)$ significantly increased over the time period of 8 hours (mean \pm SEM 7.6-fold \pm 3.1 -fold; $P=.009$ ) (Figure 1A). In contrast, mRNA levels of IFN- $\gamma$ slightly decreased when NK cells were incubated alone ( 0.2 -fold \pm 0.1 -fold; ns). As a result, at time point 8 hours, transcript levels of IFN- $\gamma$ were 38fold higher in NK cells coincubated with the fungus $(P$
$=.036)($ Figure 1A and Table 1). However, despite the increased gene expression, the protein levels of IFN- $\gamma$ measured after 8 hours in the supernatant of NK cells co-incubated with $A$. fumigatus were slightly lower than those of NK cells incubated alone (mean \pm SEM: $74 \pm 33$ $\mathrm{pg} / \mathrm{mL}$ versus $97 \pm 49 \mathrm{pg} / \mathrm{mL}$ ) (Figure $1 \mathrm{~B}$ and Table 1). To further investigate this phenomenon, we assessed the level of translation of IFN- $\gamma$. Western blot analysis revealed that the translation of IFN- $\gamma$ occurred, but that the presence of A. fumigatus resulted in a significant intracellular accumulation of IFN- $\gamma($ mean \pm SEM of the IFN- $\gamma /$ GAPDH ratio $4 \pm 1.1$ in NK cells incubated with the fungus versus $1.0 \pm 0.15$ in NK cells incubated alone; $P=.0106$ ) (Figure 1C). In contrast, when A. fumigatus in different concentrations was added to IFN- $\gamma$, the protein concentration was not affected, suggesting that fungal proteases do not play a major role in the low levels of IFN- $\gamma$ in the supernatant (data not shown).
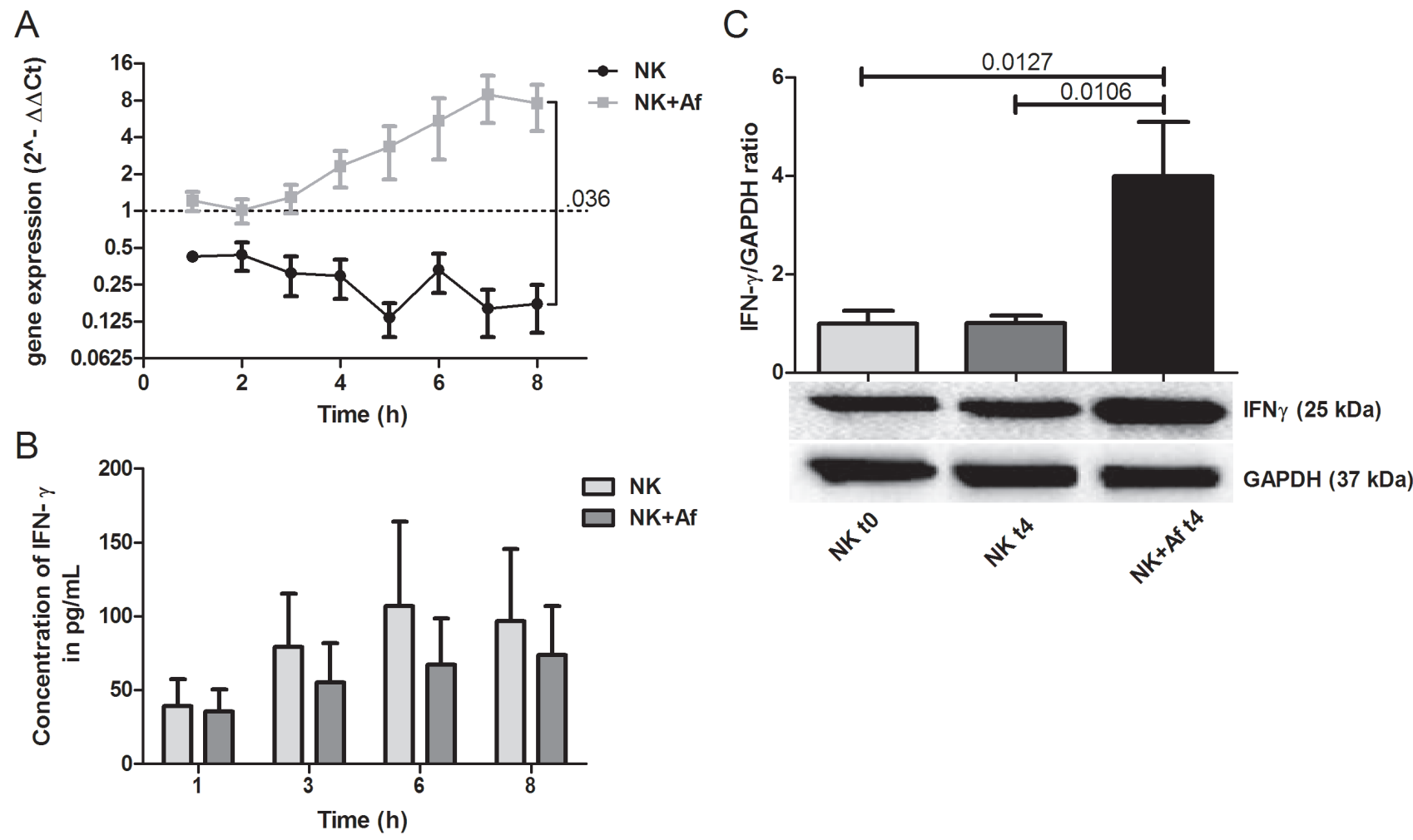

Figure 1: Gene expression and intra- and extracellular protein levels of IFN- $\boldsymbol{\gamma}$. A. Gene expression of interferon-gamma (IFN- $\gamma ; I F N G$ ) in interleukin (IL)-2 pre-stimulated human NK cells co-incubated with A. fumigatus hyphae (grey squares, NK+Af) or incubated alone (black dots, NK). The $\mathrm{X}$ axis represents the time (hours); first assessment of transcript levels was performed at hour 1. The $\mathrm{Y}$ axis represents the relative fold-change of IFN- $\gamma$ at specific time points to IFN- $\gamma$ at time point 0 (dotted line; $<1$ down-regulation, $>1$ upregulation). Squares and dots represent means, bars the standard error of means $(n=5)$. The $P$ value represents the difference at time point 8 hours. B. Concentration of IFN- $\gamma$ in the supernatant of NK cells incubated for up to 8 hours with A. fumigatus hyphae (dark-grey columns on the right, $\mathrm{NK}+\mathrm{Af}$ )) or without the fungus (light-grey columns on the left, NK). The boxes represent means, the whiskers standard error $(n=5)$. C. Bottom: Western blot analysis of intracellular IFN- $\gamma$ and GAPDH in NK cells incubated with A. fumigatus hyphae (NK + Af t4) or without the fungus (NK t4) for 4 hours and of the control at time point 0 (NK t0). Shown is one representative experiment out of a total of five independent experiments. Top: Calculated ratios of IFN- $\gamma$ to GAPDH in NK cells at 0 hours (light-grey column on the left), NK cells incubated alone for 4 hours (dark-grey column in the center) and NK cells incubated with A. fumigatus hyphae for 4 hours (black column on the right). The boxes represent means, the whiskers standard error $(n=5)$. 
Table 1: Regulation of selected genes and extracellular protein levels of human Natural Killer cells in the presence or absence of Aspergillus fumigatus.

\begin{tabular}{|c|c|c|c|c|c|c|}
\hline \multirow[b]{2}{*}{$\begin{array}{l}\text { human NK } \\
\text { cells }\end{array}$} & \multirow[b]{2}{*}{ gene (molecule) } & \multicolumn{3}{|c|}{ gene expression } & \multicolumn{2}{|l|}{ protein level } \\
\hline & & \begin{tabular}{|l|l|}
$x-$ fold \\
change
\end{tabular} & $2^{-\Delta \Delta \mathrm{Ct}} ; \mathrm{NK} / \mathrm{NK}+\mathrm{Af}$ & $P$ & $\mathrm{pg} / \mathrm{mL} ; \mathrm{NK} / \mathrm{NK}+\mathrm{Af}$ & $P$ \\
\hline & Cytotoxic molecules & & & & & \\
\hline & $P R F 1$ (perforin) & -3 & $2.1 \pm 0.1 / 0.7 \pm 0.1$ & $<0.0001$ & $\begin{array}{l}16229 \pm 1814 / 17355 \pm \\
1302\end{array}$ & ns \\
\hline & GZMB (granzyme B) & -2.6 & $0.8 \pm 0.1 / 0.3 \pm 0.1$ & 0.001 & $2752 \pm 544 / 4172 \pm 314$ & ns \\
\hline & Pro-inflammatory molecules & & & & & \\
\hline & IFNG (interferon-gamma) & +38 & $0.2 \pm 0.1 / 7.6 \pm 3.1$ & 0.036 & $97 \pm 49 / 74 \pm 33$ & $\mathrm{~ns}$ \\
\hline & $G M C S F$ (GM-CSF) & +4.6 & $0.7 \pm 0.1 / 3.2 \pm 0.7$ & 0.015 & $29 \pm 14 / 28 \pm 11$ & ns \\
\hline & $\begin{array}{l}\text { MIPIA (macrophage inflammatory protein } \\
1 \alpha)\end{array}$ & +3.7 & $0.6 \pm 0.1 / 2.2 \pm 0.5$ & 0.016 & $1900 \pm 768 / 1891 \pm 784$ & ns \\
\hline & $\begin{array}{l}\text { MIPIB (macrophage inflammatory protein } \\
1 \beta)\end{array}$ & +3.7 & $0.7 \pm 0.1 / 2.6 \pm 0.7$ & 0.044 & $2402 \pm 805 / 1923 \pm 448$ & ns \\
\hline
\end{tabular}

Relative change of the mRNA expression of the gene of interest after 8 hours of (co-) incubation relative to the housekeeping gene and to time point at hour 0 , respectively. The first value represents the results in NK cells incubated alone (NK), the second value the results in NK cells incubated with the fungus (NK+Af) (mean $\pm \mathrm{SEM}$ each). Negative values of " $\mathrm{x}$-fold change" indicate down-regulation, positive values up-regulation.

Protein levels in the supernatant of the molecule of interest were assessed after 8 hours. The first value represents the results in NK cells incubated alone (NK), the second value the results in NK cells incubated with the fungus (NK+Af) (mean $\pm \mathrm{SEM}$ each). Shown are the results of at least five independent experiments.

A

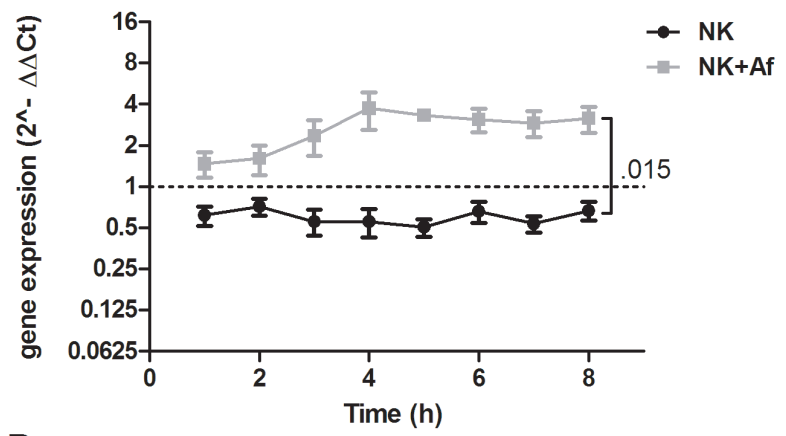

$\mathrm{B}$

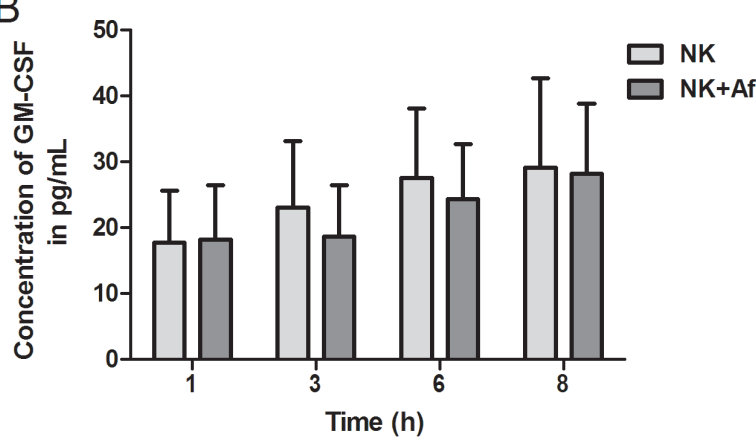

C

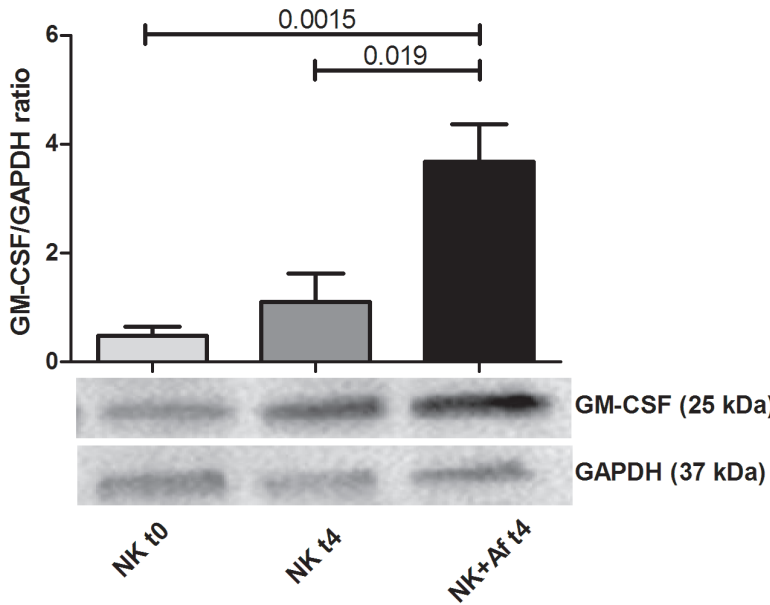

Figure 2: Gene expression and intra- and extracellular protein levels of GM-CSF. A. Gene expression of granulocytemacrophage colony stimulating factor (GM-CSF; GMCSF) in interleukin (IL)-2 pre-stimulated human NK cells co-incubated with $A$. fumigatus hyphae (grey squares, $\mathrm{NK}+\mathrm{Af}$ ) or incubated alone (black dots, $\mathrm{NK}$ ). The $\mathrm{X}$ axis represents the time (hours); first assessment of transcript levels was performed at hour 1 . The $\mathrm{Y}$ axis represents the relative fold-change of GM-CSF at specific time points to GM-CSF at time point 0 (dotted line; $<1$ down-regulation, $>1$ up-regulation). Squares and dots represent means, bars the standard error of means $(n=$ 5). The $P$ value represents the difference at time point 8 hours.B. Concentration of GM-CSF in the supernatant of NK cells incubated for up to 8 hours with A. fumigatus hyphae (dark-grey columns on the right, NK+Af)) or without the fungus (light-grey columns on the left, NK). The boxes represent means, the whiskers standard error $(n=5)$. C. Bottom: Western blot analysis of intracellular GM-CSF and GAPDH in $\mathrm{NK}$ cells incubated with $A$. fumigatus hyphae (NK $+\mathrm{Af}$ t4) or without the fungus (NK t4) for 4 hours and of the control at time point 0 (NK t0). Shown is one representative experiment out of a total of five independent experiments. Top: Calculated ratios of GM-CSF to GAPDH in NK cells at 0 hours (light-grey column on the left), NK cells incubated alone for 4 hours (dark-grey column in the center) and NK cells incubated with A. fumigatus hyphae for 4 hours (black column on the right). The boxes represent means, the whiskers standard error $(n=5)$. 
Similar results were seen for GM-CSF. Compared to NK cells coincubated alone, the presence of $A$. fumigatus resulted in 4.6-fold increased transcript levels $(P=$ $.015)$, and increased intracellular protein concentrations (mean \pm SEM of the GM-CSF/GAPDH ratio $3.7 \pm 0.7$ in NK cells incubated with the fungus versus $1.1 \pm 0.5$ in NK cells incubated alone; $P=.019)$, whereas extracellular protein concentrations did not change $(29 \pm 14 \mathrm{pg} / \mathrm{mL}$ versus $28 \pm 11$ $\mathrm{pg} / \mathrm{mL}$ ) (Figure 2 and Table 1).

Similarly, the gene expression of the proinflammatory molecules MIP-1 $\alpha$ (MIP1A) and MIP$1 \beta(M I P 1 B)$ significantly increased in the presence of A. fumigatus (3.7-fold each, $P=.016$ and .044, respectively) (Table 1). However, the up-regulation in the gene expression did not result in an increase of protein concentration in the supernatant (mean \pm SEM: $1900 \pm 768$ $\mathrm{pg} / \mathrm{mL}$ versus $1891 \pm 784 \mathrm{pg} / \mathrm{mL}$ and $2402 \pm 805 \mathrm{pg} / \mathrm{mL}$ versus $1923 \pm 448 \mathrm{pg} / \mathrm{mL}$, respectively; Table 1 ).

\section{A. fumigatus down-regulates mRNA levels of perforin in human NK cells, but increases intracellular perforin concentrations and triggers the release of perforin}

The presence of $A$. fumigatus significantly decreased the gene expression of the cytotoxic molecules perforin $(P R F 1)$ and granzyme B $(G Z M B)$ during the 8 hours of coincubation (PRF1 $P=.001 ; G Z M B P=.018)$. In contrast, mRNA levels of both cytotoxic molecules increased over time when NK cells were incubated alone ( $P=.016$ for perforin, not significant for granzyme B). As a result, mRNA levels were significantly lower in NK cells after 8 hours of coincubation with $A$. fumigatus compared to NK cells incubated alone $(P R F 1 P<.0001 ; G Z M B P=$ .001) (Figure 3A and Table 1).

Despite the decrease of the gene expression,

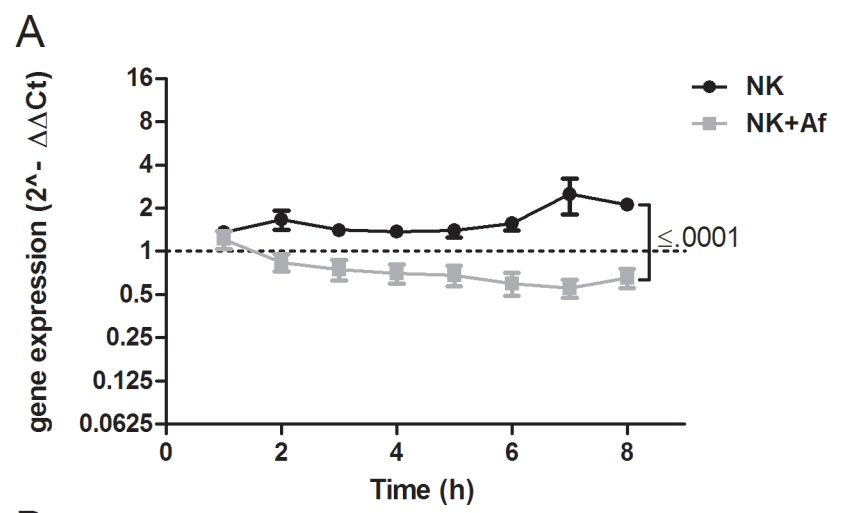

C
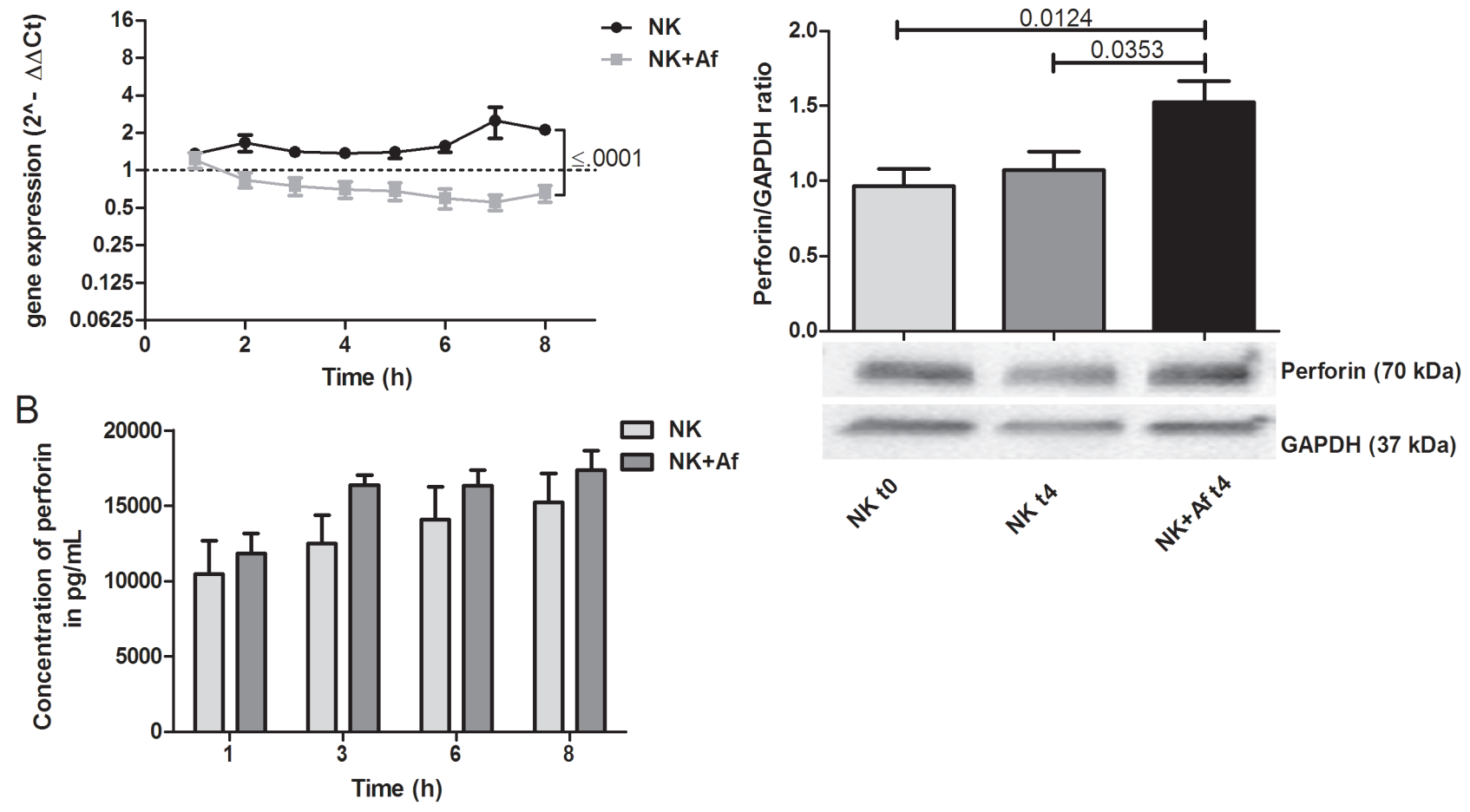

Figure 3: Gene expression and intra- and extracellular protein levels of perforin. A. Gene expression of perforin $(P R F 1)$ in interleukin (IL)-2 pre-stimulated human NK cells co-incubated with A. fumigatus hyphae (grey squares, NK+Af) or incubated alone (black dots, NK). The $\mathrm{X}$ axis represents the time (hours); first assessment of transcript levels was performed at hour 1 . The $\mathrm{Y}$ axis represents the relative fold-change of perforin at specific time points to perforin at time point 0 (dotted line; $<1$ down-regulation, $>1$ up-regulation). Squares and dots represent means, bars the standard error of means $(n=5)$. The $P$ value represents the difference at time point 8 hours.B. Concentration of perforin in the supernatant of NK cells incubated for up to 8 hours with $A$. fumigatus hyphae (dark-grey columns on the right, $\mathrm{NK}+\mathrm{Af})$ ) or without the fungus (light-grey columns on the left, NK). The boxes represent means, the whiskers standard error $(n=$ 5). C. Bottom: Western blot analysis of intracellular perforin and GAPDH in NK cells incubated with A. fumigatus hyphae (NK + Af t4) or without the fungus (NK t4) for 4 hours and of the control at time point 0 (NK t0). Shown is one representative experiment out of a total of five independent experiments. Top: Calculated ratios of perforin to GAPDH in NK cells at 0 hours (light-grey column on the left), NK cells incubated alone for 4 hours (dark-grey column in the center) and NK cells incubated with A. fumigatus hyphae for 4 hours (black column on the right). The boxes represent means, the whiskers standard error $(n=5)$. 
Table 2: Regulation of selected genes in Aspergillus fumigatus in the presence or absence of human NK cells.

\begin{tabular}{|c|c|c|c|c|}
\hline A. fumigatus & & gene expressic & & \\
\hline & gene (molecule) & $x$-fold change & $2^{-\Delta \Delta C t} ; \mathbf{A f} / \mathbf{A f}+\mathbf{N K}$ & $\bar{P}$ \\
\hline & Heat shock response & & & \\
\hline & hsp70 (heat shock protein 70) & +1.1 & $0.9 \pm 0.1 / 1.0 \pm 0.1$ & $\mathrm{~ns}$ \\
\hline & hsp90 (heat shock protein 90) & +1.6 & $1.0 \pm 0.1 / 1.6 \pm 0.1$ & 0.007 \\
\hline & High affinity iron assimilation & & & \\
\hline & fre $B$ (ferric chelate reductase) & +1.6 & $0.9 \pm 0.0 / 1.4 \pm 0.1$ & 0.001 \\
\hline & Proteases/ Peptidases & & & \\
\hline & alp1 (alkaline protease 1) & +2.1 & $0.9 \pm 0.0 / 1.9 \pm 0.3$ & 0.01 \\
\hline & $d p p I V$ (dipeptidyl peptidase IV) & +1.5 & $0.6 \pm 0.1 / 0.9 \pm 0.1$ & $\mathrm{~ns}$ \\
\hline & $d p p V$ (dipeptidyl peptidase $\mathrm{V})$ & \pm 1.0 & $1.3 \pm 0.2 / 1.3 \pm 0.2$ & ns \\
\hline & ROS $^{\text {a detoxification }}$ & & & \\
\hline & sodl (superoxide dismutase 1) & \pm 1.0 & $0.9 \pm 0.2 / 0.9 \pm 0.2$ & $\mathrm{~ns}$ \\
\hline & $\operatorname{sod} 2$ (superoxide dismutase 2$)$ & +1.4 & $0.9 \pm 0.2 / 1.3 \pm 0.3$ & $\mathrm{~ns}$ \\
\hline & sod3 (superoxide dismutase 3) & +1.1 & $1.5 \pm 0.0 / 1.6 \pm 0.2$ & ns \\
\hline & cat1 (catalase 1$)$ & \pm 1.0 & $0.6 \pm 0.1 / 0.6 \pm 0.1$ & ns \\
\hline & cat2 (catalase 2) & +1.3 & $1.3 \pm 0.0 / 1.7 \pm 0.4$ & ns \\
\hline & cycA (cytochrome $\mathrm{C})$ & \pm 1.0 & $0.8 \pm 0.1 / 0.8 \pm 0.2$ & ns \\
\hline & Mycotoxins & & & \\
\hline & $m g l$ (mitogillin) & +1.6 & $0.7 \pm 0.1 / 1.1 \pm 0.2$ & ns \\
\hline & gliT (gliotoxin) & +1.2 & $0.7 \pm 0.1 / 1.1 \pm 0.2$ & ns \\
\hline
\end{tabular}

${ }^{a} \mathrm{ROS}=$ reactive oxygen species

Relative change of the mRNA expression of the gene of interest after 8 hours of (co-) incubation relative to the housekeeping gene and to time point at hour 0 , respectively. The first value represents the results in A. fumigatus incubated alone (Af), the second value the results in $A$. fumigatus incubated with NK cells $(\mathrm{Af}+\mathrm{NK})$ (mean \pm SEM each). Negative values of "x-fold change" indicate down-regulation, positive values up-regulation. Shown are the results of at least five independent experiments.

A. fumigatus increased the protein concentration of perforin in NK cells (Figure 3C). Western blot analysis demonstrated a ratio of perforin to GAPDH of $1.5 \pm 0.14$ (mean \pm SEM) after 4 hours of coincubation with the fungus versus $0.96 \pm 0.12$ when NK cells were incubated alone $(P$ $=.0353)$. The high perforin levels in the supernatant of IL-2 pre-stimulated NK cells (mean \pm SEM $16,229 \pm 1814$ $\mathrm{pg} / \mathrm{mL}$ at time point 8 hours) were only slightly increased in the presence of A. fumigatus (mean \pm SEM 17,335 \pm 1302 $\mathrm{pg} / \mathrm{mL}$ ) (Figure 3B and Table 1).

\section{A. fumigatus up-regulates the gene expression of stress related molecules in the presence of human NK cells}

The transcript levels of the heat shock protein 90 (hsp90) were significantly higher when A. fumigatus was coincubated with NK cells ( $P=.007$ at time point 8 hours) (Figure 4A and Table 2). Similarly, transcript levels of the fungal ferric chelate reductase (freB) were significantly increased upon exposure with NK cells at all time points investigated ( $P=.001$ at time point 8 hours) (Figure 4B and Table 2).

\section{Human NK cells differentially regulate the gene expression of fungal proteases, but do not have a major impact on fungal superoxide dismutases, catalases and mycotoxins}

In the presence of human NK cells, A. fumigatus expressed significantly higher mRNA levels of alkaline phosphatase 1 (alpl) compared to those when the fungus was incubated alone ( $P=.01$ after 8 hours of coincubation) (Figure 4C and Table 2). Transcript levels of the dipeptidyl peptidases IV and V, the superoxide dismutases 1-3, the catalases 1 and 2, and the mycotoxins gliotoxin and mitogillin were not significantly affected by the presence of NK cells (Figure 4D-4F and Table 2).

\section{DISCUSSION}

Our data show that NK cells and A. fumigatus interact and mutually influence the gene expression of a variety of molecules of both host response and fungal survival pathways. A. fumigatus responds to NK cells with an up-regulation of stress related genes, whereas NK cells up-regulate in the presence of A. fumigatus 
the gene expression of pro-inflammatory cytokines such as IFN- $\gamma$ or GM-CSF. This is not surprising, as these molecules play an important role in the antifungal host response, which is in line with results previously reported in NK cells coincubated with C. albicans [9]. IFN- $\gamma$ stimulates the migration of phagocytes, enhances their phagocytic and oxidative killing activity of $A$. fumigatus, and is a signature cytokine of protective $\mathrm{T}_{\mathrm{H}} 1$ response [10]. Similarly, GM-CSF stimulates the production of professional phagocytes and enhances their antifungal activity [11]. We observed that the presence of $A$. fumigatus increases the gene expression of these molecules, which are also translated into proteins, but, at the same time, leads to an intracellular accumulation, which decreases the extracellular availability of these immunomodulators. Although we cannot exclude that there is an increased release of the proteins after our observation time period of 8 hours, it is important to note that NK represent the dominant source of IFN- $\gamma$ during the early phase of infection when the adoptive immunity is not yet active as well as in individuals with a compromised $\mathrm{T}$ cell function $[3,12]$. This immunosuppressive effect of A. fumigatus on NK cells has not been reported before, and might have deleterious consequences for the host. For example, it has been demonstrated in neutropenic mice with invasive aspergillosis that the depletion of
NK cells resulted in reduced lung IFN- $\gamma$ levels and increased lung fungal load that was independent of $\mathrm{T}$ and B cell lymphocytes [3]. However, when NK cells in IFN- $\gamma$ deficient mice were depleted, no further increase in severity of the infection was seen. Finally, the transfer of activated NK cells from wild-type, but not from IFN- $\gamma$ deficient mice led to greater pathogen clearance from the lungs of both IFN- $\gamma$ deficient and wild-type recipients, all of which underlines the importance of NK cell derived IFN- $\gamma$ in the host defense against $A$. fumigatus. The detailed analysis of this newly described effect of $A$. fumigatus, which is immunosuppressive by the intracellular accumulation of proinflammatory cytokines, is the current focus of our research and might help to develop better immunotherapeutic strategies in the future. Importantly, A. fumigatus and R. oryzae differently affect the concentration of IFN- $\gamma$ and GMCSF in the supernatant $[5,13]$, which suggests that each fungus exhibits specific immunosuppressive effects on NK cells and might offer new diagnostic tools. However, the interpretation of studies investigating the effect of fungi on NK cells has to consider whether and how the NK cells were prestimulated. Whereas we did not observe a significant difference in the gene expression profiles between unstimulated and IL-2 prestimulated NK cells (data not shown), prestimulation with other cytokines
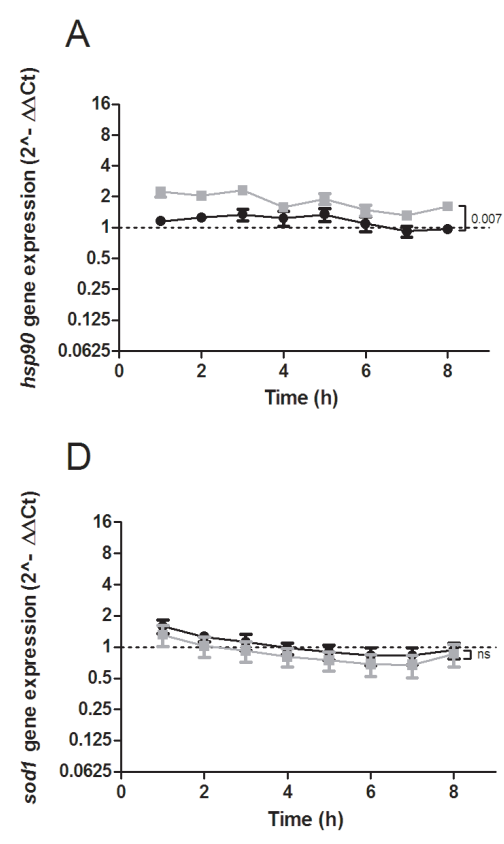
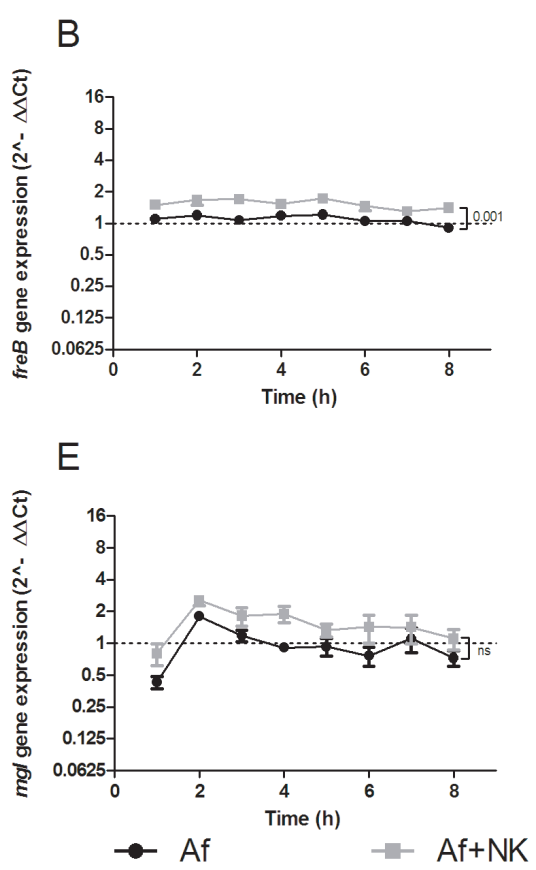

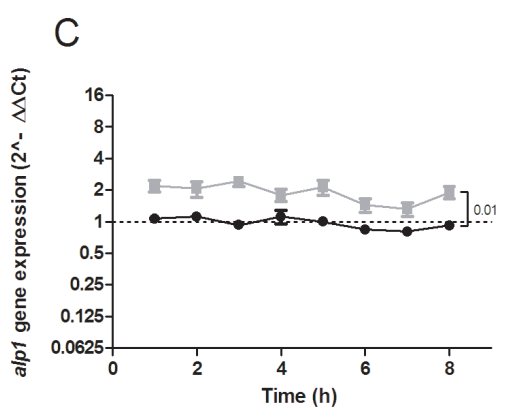

$\mathrm{F}$

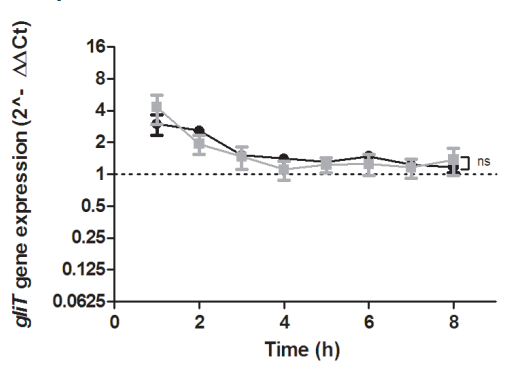

Figure 4: Gene expression profile of heat shock protein90 ( $\mathrm{sp} 90)$ A., ferric chelate reductase (freB) B., alkaline protease 1 (alp1) C., superoxide dismutase ( $\operatorname{sod} l)$ D., mitogillin $(m g l)$ E., and gliotoxin $(g l i T)$ F. assessed in A. fumigatus co-incubated with pre-stimulated human NK cells (grey squares, $\mathrm{Af}+\mathrm{NK}$ ), or incubated alone (black dots, Af). The $\mathrm{X}$ axis represents the time (hours); the first assessment of transcript levels was performed at hour 1 . The $\mathrm{Y}$ axis represents the relative fold-change ( $<1$ down-regulation, $>1$ up-regulation) of the gene of interest at specific time points relative to time point 0 (dotted line; $<1$ down-regulation, $>1$ up-regulation).. Squares and dots represent means, bars the standard error of means $(n=5)$. The $P$ value represents the difference at time point 8 hours. 
such as IL-12 or IL-18 may have different effects on NK cells [14]. Although our approach of prestimulating with IL-2 over 7-10 days is currently the standard setting for many clinical trials using NK cells, the effect of fungi on NK cells prestimulated with different protocols has to be assessed in the future $[15,16]$.

The transcript levels of perforin and granzyme B, which play a central role in NK cell mediated killing of fungi $[5,9,13]$, increase in IL-2 prestimulated NK cells in the absence of $A$. fumigatus, but decrease in the presence of the fungus. The decrease of the gene expression of perforin with a concomitant increase of the intracellular protein concentration seems surprising, but can be explained by the fact that upon activation with $A$. fumigatus, NK cells rapidly upregulate the translation of preexisting mRNA of perforin [17]. The presence of $A$. fumigatus also leads to a slight increase of the perforin levels in the supernatant due to degranulation, an effect which has also been observed in NK cells coincubated with $R$. oryzae or C. albicans [13, 18]. However, as IL-2 prestimulation of NK cells alone already results in relatively high extracellular perforin levels and enhanced cytotoxicity, A. fumigatus only had a marginal effect on the extracellular perforin concentration.

The presence of NK cells resulted in an upregulation of the expression of several stress-related fungal genes, such as the heat shock protein $h s p 90$. This is plausible, as stress response pathways are essential for adaptation to hostile environments which Aspergillus hyphae encounter when coincubated with human NK cells. Hsp90 of $A$. fumigatus is at the center of a complex network involving calcineurin, lysine deacetylases and other client proteins, which orchestrate compensatory repair mechanisms of the cell wall in response to the stress induced by antifungals [19]. Interestingly, disrupting Hsp90 circuitry by different means such as Hsp90 inhibitors potentiates the activity of the antifungal compound caspofungin [19], suggesting that further studies should investigate the influence of this approach in order to increase the efficacy of antifungal strategies. We also observed a significant increase in the gene expression of the ferric reductase fre $B$, which has recently been identified as an important enzyme in filamentous fungi for the adaptation to iron starvation [20]. Our results are in line with a recent study in $C$. albicans which reports that the perforin-induced reduction of iron availability results in the upregulation of the gene expression of Csa 2, which is involved in the uptake of iron of human hemoglobin [9]. Iron plays a key role in fungal growth and survival, and animal studies have shown a benefit of iron chelators as a therapeutic strategy in invasive aspergillosis [21].

Proteins secreted by the fungus such as peptidases and proteases play an important role in the pathogenesis of invasive fungal disease as they help to invade host tissue and to manifest invasive growth. In this respect, we observed an up-regulation of the transcript levels of the fungal alkaline protease 1 (asp f13, alp1) when the pathogen was coincubated with prestimulated human NK cells. This molecule is involved in the pathogenesis of asthma and other lung diseases associated with epithelial barrier impairment, as it infiltrates the bronchial submucosa and disrupts airway smooth muscle cellextracellular matrix interactions [22]. In addition, Alp1 plays a role in $\mathrm{T}_{\mathrm{H}} 1 / \mathrm{T}_{\mathrm{H}} 2$ differentiation, helps $A$. fumigatus to evade from the host complement attack by its complement-degrading activity and might therefore be an interesting target in the development of antifungal therapy $[23,24]$.

Interestingly, $A$. fumigatus seems to be able to specifically adapt the response to the host immune cell. For example, co-incubation of the fungus with airway epithelial cells leads to an upregulation of genes counteracting oxidative stress [25], whereas we did not observe an alteration of the gene expression of the catalases cat 1 and cat 2 in the presence of NK cells, in which reactive oxygen species do not play a major role in the clearance of pathogens.

As a number of molecules involved in the antifungal activity of NK cells have previously been described, we decided to analyze the gene expression and protein concentrations of these molecules in more detail. However, future studies analyzing the transcriptome and proteome need to complement our results characterizing the complex host-pathogen interaction.

In conclusion, we describe for the first time that A. fumigatus exhibits an immunosuppressive effect on human NK cells by inhibiting the release of proinflammatory cytokines, whereas $A$. fumigatus upregulates in the presence of NK cells stress-related genes. The results of our study might help to develop approaches to strengthen specific antifungal host immune responses, which ultimately could improve outcome of invasive aspergillosis.

\section{MATERIALS AND METHODS}

\section{Isolation and cultivation of primary human NK cells}

Primary human NK cells were isolated from peripheral blood from different healthy volunteers. Cells were isolated by negativ selection using the EasySep $^{\circledR}$ Human NK Cell Enrichment Kit (StemCell Technologies, Grenoble, France) according to the manufacturer's instructions. Viability and purity of the isolated $\mathrm{CD}^{2} 6^{+} \mathrm{CD}^{-} \mathrm{NK}$ cells were $\geq 92 \%$ and $\geq 98 \%$, respectively, as determined by flow cytometry (Canto II, Beckton Dickinson, San Jose, USA). Isolated NK cells were cultivated for 10 days in RPMI (Gibco, Paisley, UK) 
supplemented with 5\% human frozen plasma (German Red Cross Blood Donor Service Baden-Wuerttemberg-Hessen, Frankfurt, Germany) and stimulated with recombinant human interleukin (rhIL)-2 (1000 U/mL; Novartis, Basel, Switzerland) every three days. The protocol was approved by the local ethics committee.

\section{Preparation of $A$. fumigatus}

The A. fumigatus strain AF4215 (MYA 1163; American Type Culture Collection) was grown on Sabouraud glucose agar (BD Bioscience, San Jose, USA) at $37^{\circ} \mathrm{C}$ for $2-3$ days. Conidia were harvested by gently washing the surface with PBS (Gibco) supplemented with 0.05\% Tween-20 (Sigma-Aldrich, Taufkirchen, Germany). The number of the conidia was estimated in a Neubauer slide (LO-Laboroptik, Friedrichsdorf, Germany). Resting conidia were immediately used or stored at $4{ }^{\circ} \mathrm{C}$. For preparation of $A$. fumigatus hyphae, $1.25 \times 10^{4}$ resting conidia were plated in 48-well flat-bottom cell culture plates (Nunc, Langenselbold, Germany) and incubated in $500 \mu \mathrm{L}$ Yeast Nitrogen Base (Sigma-Aldrich) supplemented with (D)-Glucose (Sigma-Aldrich) at $37^{\circ} \mathrm{C}$ for 17 hours to allow formation of mycelium.

\section{Co-incubation of $A$. fumigatus and human NK cells and preparation of total RNA}

A total of $2.5 \times 10^{5} \mathrm{NK}$ cells were co-incubated with A. fumigatus mycelium in $500 \mu \mathrm{L} \mathrm{NK}$ cell culture medium in 48-well flat-bottom culture plates (Nunc) for up to 8 hours at $37^{\circ} \mathrm{C}$.

For the preparation of RNA, samples were collected immediately prior to co-incubation and thereafter every hour during co-incubation for up to 8 hours. Total RNA of both NK cells and the fungus was immediately extracted by means of the RNeasy Plus Micro Kit (Qiagen, Hilden, Germany) according to the manufacturer's instructions. For extraction of fungal RNA, the cell wall of $A$. fumigatus was cracked by three freeze-thaw cylces, consisting of heating to $95^{\circ} \mathrm{C}$ and direct freezing in liquid nitrogen to $-196^{\circ} \mathrm{C}$. The isolated RNA was dissolved in RNase-free water and the concentration and purity of the samples were determined using a NanoDrop ${ }^{\circledR}$ ND-1000 spectrophotometer (Thermo Scientific, Wilmington, USA). For the reverse transcription of the mRNA of $A$. fumigatus and NK cells to cDNA, the High Capacity RNA-to-cDNA Kit (Applied Biosystem, Foster City, USA) was used according to the manufacturer's instruction.

\section{Primers and amplicons for human NK cells}

Representative target-specific NK cell gene sequences were obtained by the NCBI GeneBank. Both primer pairs and UPL TaqMan ${ }^{\circledR}$ probes were selected from conserved regions of respective specific sequences based on the intron-spanning method of the online Universal Probe Library Assay Design Center (Roche, Mannheim, Germany). Primers were purchased from MWG (Eurofins Genomics, Ebersberg, Germany), UPL probes from Roche (Supplementary Table 1). Amplicon lenghts ranged between 66 and $123 \mathrm{bp}$ with an annealing temperature of $60^{\circ} \mathrm{C}$. Uniqueness was tested with the NCBI BLAST search.

\section{Primers and amplicons for $A$. fumigatus}

Sequences for $A$. fumigatus were retrieved from the Central Aspergillus Data REpository site [6]. Gene identities and primer sequences are listed in Supplementary Table 2. Primers were designed to span exon-intron boarders, and amplicon lengths ranged between 104 and $138 \mathrm{bp}$ with an annealing temperature of $58^{\circ} \mathrm{C}$. Primer efficiency was analyzed by ten-fold serial dilutions for human and Aspergillus specific primer-setups.

\section{Quantitative real-time PCR for human NK cells}

Real-time PCR for human NK cell genes was performed in a total volume of $20 \mu \mathrm{L}$ and for each gene analyzed in technical duplicates. The mastermix contained 1x ABsolute qPCR ROX Mix (Thermo Scientific), primer pairs and probes $250 \mathrm{nM}$ each, and 1 $\mu \mathrm{L}$ of template cDNA. The qRT-PCR was performed in a iQ5 platform (Bio-Rad, Munich, Germany) using the following thermal cycling conditions: $95^{\circ} \mathrm{C}$ for $12 \mathrm{~min}$ for initial denaturation, followed by 55 cycles of $95^{\circ} \mathrm{C}$ for $15 \mathrm{sec}$, and $60^{\circ} \mathrm{C}$ for $60 \mathrm{sec}$. All PCR assays included a housekeeping gene of human NK cells as reference [glyceraldehyde 3-phosphate dehydrogenase (GAPDH)] [7]. The Ct-values were generated by the Bio-Rad iQ5 Software with a threshold of 100 . The $2^{-\Delta \Delta \mathrm{Ct}}$-method was used to calculate the regulation of the genes [8].

\section{Quantitative real-time PCR for $A$. fumigatus}

qRT-PCR was performed from cDNA using SsoFast EvaGreen Supermix (Bio-Rad) in a Bio-Rad CFX96 real time PCR detection system. The total reaction volume was $20 \mu \mathrm{L}$, containing forward and reverse primers $400 \mathrm{nM}$ each and $2 \mu \mathrm{l}$ of cDNA template. Reactions were performed in technical duplicates. The parameters consisted of an initial denaturation at $98^{\circ} \mathrm{C}$ for $30 \mathrm{sec}$, and 40 cycles of $98^{\circ} \mathrm{C}$ for $5 \mathrm{sec}, 58^{\circ} \mathrm{C}$ for $5 \mathrm{sec}$ followed by a melt curve analysis to confirm the specificity of the PCR products. PCR assays included a housekeeping gene of $A$. fumigatus cells as reference [beta-tubulin; $(T U B B)]$. Data were analysed using the $2^{-\Delta \Delta \mathrm{Ct}}$-method [8]. 


\section{Assessment of protein concentrations in the supernatant}

Concentrations of IFN- $\gamma$ were assessed by a commercially available enzyme-linked immunoabsorbent assay (ELISA; BioLegend, San Diego, USA), of all other molecules by cytokine bead array (CBA; BD Biosciences) according to the manufacturers' instructions. The detection limit was $5.6 \mathrm{pg} / \mathrm{mL}$ for IFN- $\gamma, 40 \mathrm{pg} / \mathrm{mL}$ for granzyme B, and $10 \mathrm{pg} / \mathrm{mL}$ for all other molecules.

\section{Western blot analysis}

Prestimulated human NK cells were coincubated with or without hyphae of $A$. fumigatus for 4 hours. NK cells at time point 0 hours served as control. Cells were lysed with RIPA Lysis and Extraction Buffer Kit (Thermo Scientific) containing HALT Phosphatase and Protease Inhibitor Cocktails (both Thermo Scientific). Proteins were separated by SDS-PAGE using 4-15\% Mini Protean TGX Precast Protein Gels (Bio-Rad). Proteins were blotted onto a nitrocellulose membrane which was blocked with 5\% skim milk-PBS solution. Mouse monoclonal antibodies against human IFN- $\gamma$ (1:200; LifeSpan BioSciences, Seattle, USA), human perforin $(1: 1000$, LifeSpan BioScience), human GM-CSF (1:1000, R\&D Systems), and human GAPDH (1:20000; Biolegend) were used as primary antibodies, goat anti-mouse IgG-HRP antibody (1: 100000; Abcam, Cambridge, UK) as secondary antibody. For visualization, Gel Doc ${ }^{\mathrm{TM}} \mathrm{XR}+$ System (Bio-Rad) and Amersham ECL Select Western Blotting Detection Reagent (GE Healthcare Life Sciences, Little Chalfont, UK). For comparison of intracellular protein levels, the ratio of the protein of interest and GAPDH was calculated by determination of relative band intensities using Image Lab 5.0 software (Bio-Rad).

\section{Statistical analysis}

Data were analyzed using GraphPad Prism (version 5.04 for Windows, GraphPad Software, La Jolla California, USA). The unpaired $t$-test was used to compare different groups. A $P$-value (two-tailed) of $<.05$ was considered to be statistically significant.

\section{CONFLICTS OF INTEREST}

The authors declare no conflicts of interest.

\section{FUNDING INFORMATION}

This work was supported by research funding from the Madeleine Schickedanz KinderKrebs Stiftung to TL.

\section{Author Contributions}

All authors designed the study; A.S., M.B., W.P., R.S., and S.S. did the experiments; A.S., M.B., W.P., and S.S. analyzed the data; C.L-F., S.S., and T.L. supervised the study; A.S., M.B., C.L-F., S.S., and T.L. wrote the manuscript; all authors read and approved the manuscript.

\section{Editorial note}

This paper has been accepted based in part on peerreview conducted by another journal and the authors' response and revisions as well as expedited peer-review in Oncotarget.

\section{REFERENCES}

1. Wood SM, Ljunggren HG and Bryceson YT. Insights into NK cell biology from human genetics and disease associations. Cell Mol Life Sci. 2011; 68:3479-3493.

2. Schmidt S, Zimmermann SY, Tramsen L, Koehl U and Lehrnbecher T. Natural killer cells and antifungal host response. Clin Vaccine Immunol. 2013; 20:452-458.

3. Park SJ, Hughes MA, Burdick M, Strieter RM and Mehrad B. Early NK cell-derived IFN-\{gamma\} is essential to host defense in neutropenic invasive aspergillosis. J Immunol. 2009; 182:4306-4312.

4. Abad A, Fernandez-Molina JV, Bikandi J, Ramirez A, Margareto J, Sendino J, Hernando FL, Ponton J, Garaizar $\mathrm{J}$ and Rementeria A. What makes Aspergillus fumigatus a successful pathogen? Genes and molecules involved in invasive aspergillosis. Rev Iberoam Micol. 2010; 27:155182.

5. Schmidt S, Tramsen L, Hanisch M, Latge JP, Huenecke $\mathrm{S}$, Koehl U and Lehrnbecher T. Human natural killer cells exhibit direct activity against Aspergillus fumigatus hyphae, but not against resting conidia. J Infect Dis. 2011; 203:430435 .

6. Mabey JE, Anderson MJ, Giles PF, Miller CJ, Attwood TK, Paton NW, Bornberg-Bauer E, Robson GD, Oliver SG and Denning DW. CADRE: the Central Aspergillus Data REpository. Nucleic Acids Res. 2004; 32:D401-405.

7. Kaszubowska L, Wierzbicki PM, Karsznia S, Damska M, Slebioda TJ, Foerster J and Kmiec Z. Optimal reference genes for qPCR in resting and activated human NK cellsFlow cytometric data correspond to qPCR gene expression analysis. J Immunol Methods. 2015; 422:125-129.

8. Schmittgen TD and Livak KJ. Analyzing real-time PCR data by the comparative C(T) method. Nat Protoc. 2008; 3:1101-1108.

9. Hellwig D, Voigt J, Bouzani M, Loffler J, Albrecht-Eckardt D, Weber M, Brunke S, Martin R, Kurzai O and Hunniger K. Candida albicans Induces Metabolic Reprogramming 
in Human NK Cells and Responds to Perforin with a Zinc Depletion Response. Front Microbiol. 2016; 7:750.

10. Lehrnbecher T, Kalkum M, Champer J, Tramsen L, Schmidt $\mathrm{S}$ and Klingebiel T. Immunotherapy in invasive fungal infection-focus on invasive aspergillosis. Curr Pharm Des. 2013; 19:3689-3712.

11. Roilides E, Holmes A, Blake C, Venzon D, Pizzo PA and Walsh TJ. Antifungal activity of elutriated human monocytes against Aspergillus fumigatus hyphae: enhancement by granulocyte-macrophage colonystimulating factor and interferon-gamma. J Infect Dis. 1994; 170:894-899.

12. Harrington L, Srikanth CV, Antony R, Shi HN and Cherayil BJ. A role for natural killer cells in intestinal inflammation caused by infection with Salmonella enterica serovar Typhimurium. FEMS Immunol Med Microbiol. 2007; 51:372-380.

13. Schmidt S, Tramsen L, Perkhofer S, Lass-Florl C, Hanisch M, Roger F, Klingebiel T, Koehl U and Lehrnbecher T. Rhizopus oryzae hyphae are damaged by human natural killer (NK) cells, but suppress NK cell mediated immunity. Immunobiology. 2013; 218:939-944.

14. Agaugue S, Marcenaro E, Ferranti B, Moretta L and Moretta A. Human natural killer cells exposed to IL-2, IL12 , IL-18, or IL-4 differently modulate priming of naive $\mathrm{T}$ cells by monocyte-derived dendritic cells. Blood. 2008; 112:1776-1783.

15. Stern M, Passweg JR, Meyer-Monard S, Esser R, Tonn T, Soerensen J, Paulussen M, Gratwohl A, Klingebiel T, Bader P, Tichelli A, Schwabe D and Koehl U. Pre-emptive immunotherapy with purified natural killer cells after haploidentical SCT: a prospective phase II study in two centers. Bone Marrow Transplant. 2013; 48:433-438.

16. Becker PS, Suck G, Nowakowska P, Ullrich E, Seifried E, Bader P, Tonn T and Seidl C. Selection and expansion of natural killer cells for NK cell-based immunotherapy. Cancer Immunol Immunother. 2016; 65:477-484.

17. Fehniger TA, Cai SF, Cao X, Bredemeyer AJ, Presti RM, French AR and Ley TJ. Acquisition of murine NK cell cytotoxicity requires the translation of a pre-existing pool of granzyme B and perforin mRNAs. Immunity. 2007; 26:798811.
18. Voigt J, Hunniger K, Bouzani M, Jacobsen ID, Barz D, Hube B, Loffler J and Kurzai O. Human natural killer cells acting as phagocytes against Candida albicans and mounting an inflammatory response that modulates neutrophil antifungal activity. J Infect Dis. 2014; 209:616626.

19. Lamoth F, Juvvadi PR and Steinbach WJ. Heat shock protein 90 (Hsp90): A novel antifungal target against Aspergillus fumigatus. Crit Rev Microbiol. 2014:1-12.

20. Blatzer M, Binder $U$ and Haas $H$. The metalloreductase FreB is involved in adaptation of Aspergillus fumigatus to iron starvation. Fungal Genet Biol. 2011; 48:1027-1033.

21. Leal SM, Jr., Roy S, Vareechon C, Carrion S, Clark H, Lopez-Berges MS, Di Pietro A, Schrettl M, Beckmann N, Redl B, Haas H and Pearlman E. Targeting iron acquisition blocks infection with the fungal pathogens Aspergillus fumigatus and Fusarium oxysporum. PLoS Pathog. 2013; 9:e1003436.

22. Balenga NA, Klichinsky M, Xie Z, Chan EC, Zhao M, Jude J, Laviolette M, Panettieri RA, Jr. and Druey KM. A fungal protease allergen provokes airway hyper-responsiveness in asthma. Nat Commun. 2015; 6:6763.

23. Behnsen J, Lessing F, Schindler S, Wartenberg D, Jacobsen ID, Thoen M, Zipfel PF and Brakhage AA. Secreted Aspergillus fumigatus protease Alp1 degrades human complement proteins C3, C4, and C5. Infect Immun. 2010; 78:3585-3594.

24. Cramer RA, Rivera A and Hohl TM. Immune responses against Aspergillus fumigatus: what have we learned? Curr Opin Infect Dis. 2011; 24:315-322.

25. Oosthuizen JL, Gomez P, Ruan J, Hackett TL, Moore MM, Knight DA and Tebbutt SJ. Dual organism transcriptomics of airway epithelial cells interacting with conidia of Aspergillus fumigatus. PLoS One. 2011; 6:e20527. 